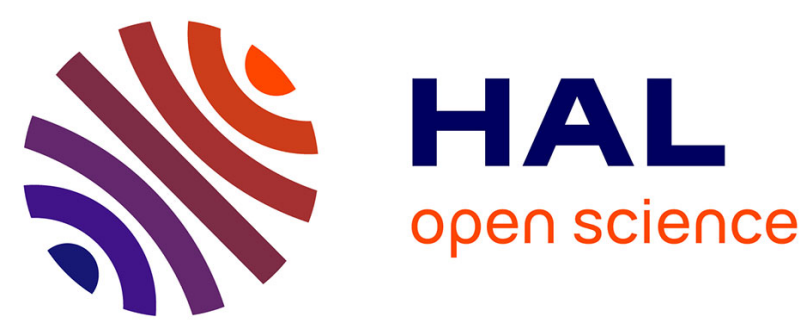

\title{
Near and far field simulation of RATAN-600 radio telescope antenna using the MLPO algorithm
}

Christine Letrou, Christian Parrot, Vladimir B. Khaikin, M. K. Lebedev, Amir Boag

\section{- To cite this version:}

Christine Letrou, Christian Parrot, Vladimir B. Khaikin, M. K. Lebedev, Amir Boag. Near and far field simulation of RATAN-600 radio telescope antenna using the MLPO algorithm. COMCAS 2011 : 3rd International Conference on Microwaves, Communications, Antennas and Electronics Systems, Nov 2011, Tel Aviv, Israel. pp.1 - 2, 10.1109/COMCAS.2011.6105931 . hal-01298131

\section{HAL Id: hal-01298131 https://hal.science/hal-01298131}

Submitted on 5 Apr 2016

HAL is a multi-disciplinary open access archive for the deposit and dissemination of scientific research documents, whether they are published or not. The documents may come from teaching and research institutions in France or abroad, or from public or private research centers.
L'archive ouverte pluridisciplinaire HAL, est destinée au dépôt et à la diffusion de documents scientifiques de niveau recherche, publiés ou non, émanant des établissements d'enseignement et de recherche français ou étrangers, des laboratoires publics ou privés. 


\title{
Near and Far Field Simulation of RATAN-600 Radio Telescope Antenna Using the MLPO Algorithm
}

\author{
C. Letrou ${ }^{1 *}$, C. Parrot ${ }^{1 \#}$, V. Khaikin ${ }^{2}$, M. Lebedev ${ }^{2}$, and Amir Boag ${ }^{3}$ \\ ${ }^{1}$ Institut Telecom, TELECOM SudParis, ${ }^{(*)}$ CNRS Lab. SAMOVAR, ${ }^{(\#)}$ Computer Science Dpt., 9 rue Charles \\ Fourier, 91011 Evry Cedex, France, email: christine.letrou@it-sudparis.eu, christian.parrot@it-sudparis.eu \\ ${ }^{2}$ The Special Astrophysical Observatory, RAS, Russia, e-mail: vkhstu@mail.ru, mike.lebedev@mail.ru \\ ${ }^{3}$ School of Electrical Engineering, Tel Aviv University, Tel Aviv 69978, Israel
}

\begin{abstract}
A fast and adaptive near-field physical optics algorithm is developed for the analysis of general multi-reflector antenna systems. The algorithm is applied to the radiation pattern computation of an extremely large antenna of RATAN600 radio telescope operating at $\mathrm{mm}$-wave frequencies. Near-field reflector to reflector field propagation and far-field radiation pattern computation results will be presented and compared with those obtained by geometrical optics with aperture integration.
\end{abstract}

Index Terms - Reflector antenna, Radio telescope, Electromagnetic modeling, High- frequency asymptotics, Physical optics.

\section{INTRODUCTION}

The RATAN-600 antenna is a multi-reflector system characterized by very large electrical dimensions. The optical scheme of the "South sector primary reflector + Flat reflector" antenna system as well as the enlarged geometry of the secondary and tertiary (auxiliary) reflectors of RATAN-600 radiotelescope are shown in Fig. 1.

Analysis of such systems at mm-wave frequencies presents a substantial computational challenge since various reflectors measure thousands of wavelengths in size. This is especially true when repeated evaluation of the radiation patterns is required for antenna geometry optimization with multiple primary sources, for example, when employing a $10 \times 10$ focal array feed. Until recently, this quasi-optical system has been mainly analyzed using Geometrical Optics (GO)/ray-tracing techniques combined with the aperture integration method [1]. For this system, even the use of GO requires substantial computational resources. On the other hand, GO analysis does not provide sufficient information on near-field diffraction effects and other wave related phenomena. Also, the aperture integration method may only be used for calculating the main beam and the nearest sidelobes. At this stage, the use of numerically rigorous integral or differential equation-based techniques that could address these concerns for problems of such large dimensions is still out of reach of existing computers.

In this context, the Physical Optics (PO) approximation appears to strike the balance between the computational feasibility and accuracy requirements while still requiring excessively long computation times in its straightforward implementation. To that end, we adapt the Multilevel
Physical Optics (MLPO) algorithm [2] for the computation of the radiation characteristics of the RATAN-600 antenna as will be described below.

\section{MLPO ALGORITHMIC DEVELOPMENTS}

The MLPO algorithm originally presented in [2] is based on the observation that small radiating apertures produce smooth radiation patterns, which can be sampled on very coarse grids and fully reconstructed by interpolation. Thus, the MLPO algorithm starts with a hierarchical decomposition of radiating surfaces into small patches, whose radiation patterns can be described on a coarse grid. Each of these patterns is interpolated in a patch-centered coordinate system allowing for slow phase variation of the fields. After translation to a common coordinate system, the field patterns of neighboring patches can be aggregated resulting in the radiation pattern of a larger patch. This sequence of operations is repeated until the pattern of the whole radiating surface is obtained.

This algorithm provides orders of magnitude acceleration of the PO integral evaluation, because it reduces the computation complexity from $O\left(N^{4}\right)$ to $O\left(N^{2} \log N\right)$ ( $N=k R$, with $R$ the radius of the smallest circumscribing sphere and $k$ the wavenumber). The MLPO algorithm achieves a computational efficiency similar to that of the FFT-based algorithms, while not being subject to their limitations (uniform sampling on planar apertures). The algorithm has been further developed in [3] to analyze dual reflector antennas.

The following extensions of the original MLPO scheme are developed to facilitate the analysis of the RATAN-600 antenna:

a) Generic multi-reflector computational scheme is developed for sequential propagation between the reflectors and aggregation of the radiation patterns produced by all system components.

b) The multilevel decomposition scheme is modified to allow for adaptive decomposition along dimensions that are substantially different in their sizes.

c) The computational sequence is parallelized in two ways: (1) under MPI for distributed memory 
clusters and (2) for graphic Processing Units (GPUs).

d) Large electrical dimensions lead to a large number of interpolation and aggregation levels incurring gradual error accumulation. To that end, error levels are verified in the computational sequence.

These extensions make the MLPO algorithm adaptive to arbitrary shaped multi-reflector antennas of very large dimensions.

\section{NUMERICAL RESULTS:}

Radiation patterns of the multi-beam RATAN-600 antenna obtained using the adaptive MLPO algorithm will be presented at the conference in comparison with the ones calculated by the combined GO-aperture integration method.

\section{REFERENCES}

[1] M. K. Lebedev, V. B. Khaikin, A. Boag, and C. Letrou, "Optical and diffraction simulation techniques for large multibeam reflector, MSMW'10: Seventh IEEE International Kharkov Symposium on Physics and Engineering of Microwaves, Millimeter and Submillimeter Waves, Kharkiv, Ukraine, pp. 1-3, June 21-26, 2010.

[2] A. Boag and C. Letrou, "Multilevel Fast Physical Optics algorithm for radiation from non-planar apertures," IEEE Trans. Antennas Propagat., vol. 53, no. 6, pp. 2064-2072, June 2005.

[3] C. Letrou and A. Boag, "Analysis of very large dual-reflector antennas using Multilevel Physical Optics algorithm," ICEAA 2007: International Conference on Electromagnetics in Advanced Applications, Turin, Italy, Sept. 2007.

(a)

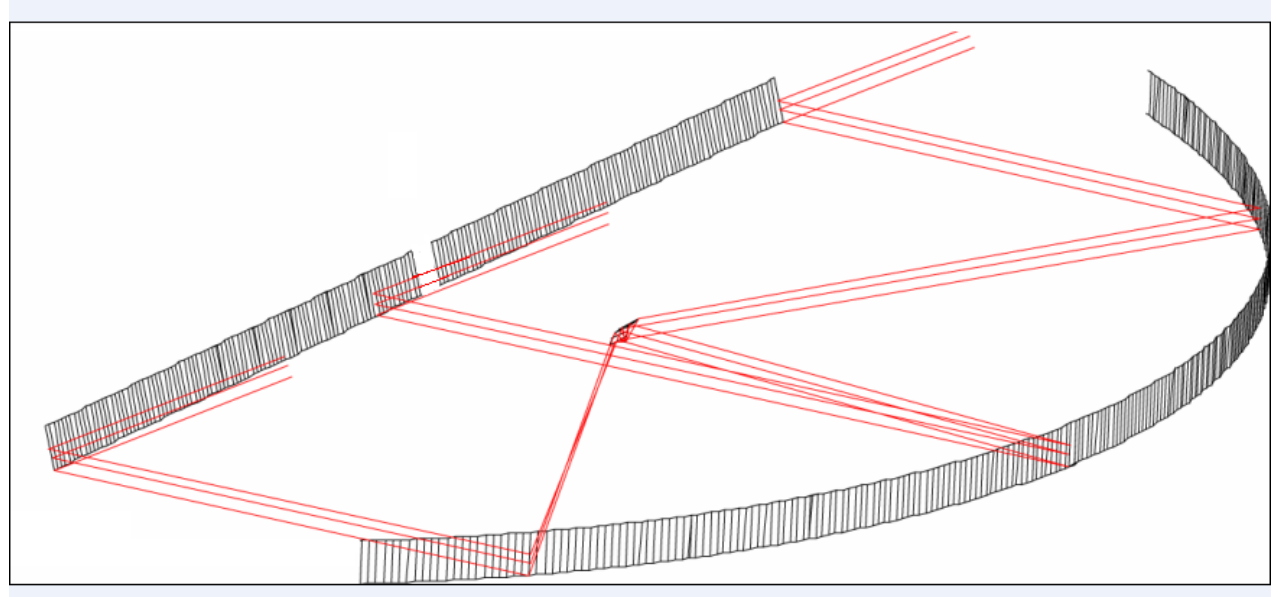

(b)

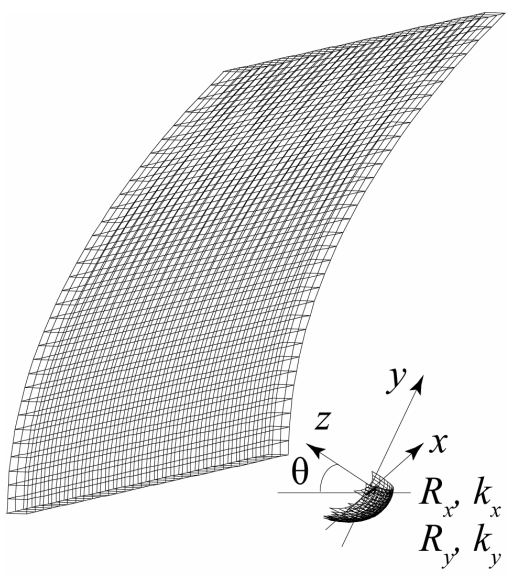

Fig. 1. (a) Optical scheme of the "South sector primary reflector + Flat reflector" antenna system and (b) geometry of the secondary and tertiary reflectors. 